\title{
An Extension of the Kumaraswamy Distribution
}

\author{
Jalmar M. F. Carrasco ${ }^{1} \&$ Gauss M Cordeiro ${ }^{2}$ \\ ${ }^{1}$ Statistics Department, Federal University of Bahia, Bahia, Brazil \\ ${ }^{2}$ Statistics Department, Federal University of Pernanbuco, Recife, Brazil \\ Correspondence: Jalmar M. F. Carrasco, Departamento de Estatistica, Instituto de Matematica e Estatistica, Universidade \\ Federal da Bahia, Salvador, BA, CEP:40.170-110, Brasil. E-mail: carrasco.jalmar@ufba.br
}

Received: December 23, 2016 Accepted: February 20, 2017 Online Published: April 11, 2017

doi:10.5539/ijsp.v6n3p61 URL: https://doi.org/10.5539/ijsp.v6n3p61

\begin{abstract}
We propose and study a new five-parameter continuous distribution in the unit interval through a specific probability integral transform. The new distribution, under some parameter constraints, is an identified parametric model that includes as special cases six important models such as the Kumaraswamy and beta distributions. We obtain ordinary and incomplete moments, quantile and generating functions, mean deviations, Rényi entropy and moments of order statistics. The estimation of the model parameters is performed by maximum likelihood, and hypothesis tests are discussed. Additionally, through a simulation study we investigate the behavior of the maximum likelihood estimator, also we investigate the impact of ignoring identifiability problems. The usefulness of the proposed distribution is illustrated by means of a real data set.
\end{abstract}

Keywords: continuous proportion; identification problems; Kumaraswamy distribution; maximum likelihood function.

\section{Introduction}

We define and study a new five-parameter distribution called the extended Kumaraswamy (EKw) distribution that includes, as special models, some well-known distributions such as the Kumaraswamy (Kw, for short) and beta ( $\mathfrak{B})$ distributions. The EKw distribution allows us to obtain new three- and four-parameter generalizations of such distributions that can be used in a variety of problems for modelling real continuous proportional data owing to its flexibility in accommodating different forms of density functions.

Wahed (2006) and Ferreira and Steel (2006) demonstrated that any parametric family of distributions can be incorporated into larger families through an application of the probability integral transform. Specifically, let $G_{1}(x ; \omega)$ be a cumulative distribution function (cdf) with associated probability density function (pdf) $g_{1}(x ; \omega)$ and $g_{2}(x ; \boldsymbol{\tau})$ be a pdf having a support over the standard unit interval. Here, $\boldsymbol{\omega}$ and $\tau$ represent scalar or vector parameters. We define

$$
F(x ; \boldsymbol{\omega}, \boldsymbol{\tau})=\int_{0}^{G_{1}(x ; \boldsymbol{\omega})} g_{2}(t ; \tau) d t
$$

where the cdf $F(x ; \omega, \tau)$ and $G_{1}(x ; \omega)$ have the same support. The pdf corresponding to (1) is given by

$$
f(x ; \boldsymbol{\omega}, \boldsymbol{\tau})=g_{2}\left(G_{1}(x ; \boldsymbol{\omega}) ; \boldsymbol{\tau}\right) g_{1}(x ; \boldsymbol{\omega}) .
$$

This mechanism for generating distributions from equation (1) is particularly attractive when $G_{1}(x ; \omega)$ has a closed-form expression. On the other hand, the beta density is often used in place of $g_{2}(x ; \tau)$. However, different choices for $G_{1}(x ; \omega)$ have been considered in the literature. Eugene et al. (2002) defined the beta normal distribution by taking $G_{1}(x ; \omega)$ as the standard normal cdf and derived some of its first moments. More general expressions for these moments were obtained by Gupta and Nadarajah (2004b). Nadarajah and Kotz (2004) defined the beta Gumbel distribution by taking $G_{1}(x ; \omega)$ to be the Gumbel cdf and provided explicit expressions for the moments and the asymptotic distribution of the extreme order statistics. Nadarajah and Gupta (2004) introduced the beta Fréchet distribution by taking $G_{1}(x ; \omega)$ to be the Fréchet distribution, derived the analytical shapes of its density and hazard rate functions, and obtained the asymptotic distribution of its extreme order statistics. Further, Nadarajah and Kotz (2006) dealt with the beta exponential distribution and determined its generating function, the first four cumulants, and the asymptotic distribution of the extreme order statistics.

The starting point of our proposal is the Kumaraswamy (Kw) distribution Jones (2009); Kumaraswamy (1980) that is quite similar to the beta distribution. It is also termed the "minimax distribution". Its closed-form cumulative function is

$$
G_{1}(x ; \omega)=1-\left(1-x^{\alpha}\right)^{\beta}, \quad 0<x<1,
$$


whereas its pdf is

$$
g_{1}(x ; \omega)=\alpha \beta x^{\alpha-1}\left(1-x^{\alpha}\right)^{\beta-1}, \quad 0<x<1,
$$

where $\boldsymbol{\omega}=(\alpha, \beta)^{\top}, \alpha>0$, and $\beta>0$. If $X$ is a random variable with density (4), we write $X \sim \operatorname{Kw}(\alpha, \beta)$. This distribution was originally conceived to model hydrological phenomena, but it has been used for other purposes(Sundar \& Subbiah, 1989; Fletcher \& Ponnambalam, 1996; Seifi, et al, 2000; Ganji, et al, 2006; Sanchez, et al, 2007; Courard-Hauri, 2007).

We propose an extension of the Kw distribution by taking $G_{1}(x ; \omega)$ as the cdf (3) and $g_{2}(x ; \tau)$ as the generalized beta density of the first kind (GB1) Gupta and Nadarajah (2004a); McDonald (1984) defined by

$$
g_{2}(x ; \tau)=\frac{\lambda}{B(\gamma, \eta)} x^{\lambda \gamma-1}\left(1-x^{\lambda}\right)^{\eta-1}, 0<x<1,
$$

where $\tau=(\gamma, \eta, \lambda)^{\top}, \gamma>0, \eta>0$, and $\lambda>0, B(\gamma, \eta)=\Gamma(\gamma) \Gamma(\eta) / \Gamma(\gamma+\eta)$ is the beta function and $\Gamma(\cdot)$ is the gamma function. If $X$ is a random variable with pdf (5), we write $X \sim \operatorname{GB} 1(\gamma, \eta, \lambda)$. Further, if $X \sim \operatorname{GB} 1(\gamma, \eta, 1)$, then $X \sim \mathfrak{B}(\gamma, \eta)$, i.e., $X$ has a beta distribution with parameters $\gamma$ and $\eta$. If $X \sim \mathrm{GB} 1(1, \eta, \lambda)$, then $X \sim \operatorname{Kw}(\eta, \lambda)$.

Remark 1 A comment on the choices of $G_{1}$ and $G_{2}$ seems in order. We consider the Kumaraswamy cdf for $G_{1}$ because it has a closed-form and it is a very competitive model for the beta distribution. For the choice of the standard generalized beta density of the first kind to $G_{2}$, the motivation has been to consider a distribution which, combined with the Kw distribution, generates a wider family, including, as special models, several known distributions. In Section 3, we show that the choice for $G_{2}$ is very successful in this regard.

The paper is organized as follows. In Section 2, we define the EKw distribution, plot its pdf for selected parameter values, and provide some of its basic structural properties. In Section 3, we present some special models. In Section 4, we demonstrate that the EKw density function can be expressed as linear combinations of Kw and power density functions. In Section 5, we determine explicit expressions for the ordinary and incomplete moments, generating function, mean deviations, Bonferroni and Lorenz curves. In Section 6, we derive the density function of the order statistics and their moments. The Rényi entropy is investigated in Section 7. In Section 8, we discuss maximum likelihood estimation and obtain the observed information matrix. Section 9 is devoted to a simulation study to prove empirically the adequacy of the maximum likelihood estimators (MLEs). In Section 10, we give an application to a real data set. Section 11 provides some conclusions.

\section{An Extension}

We obtain an appropriate extension of the $\mathrm{Kw}$ distribution by taking $g_{2}(x ; \boldsymbol{\tau})$ as the GB1 density $(5)$ and $G_{1}(x ; \boldsymbol{\omega})$ as the $\mathrm{Kw}$ cdf (3). Following (1), the five-parameter EKw cumulative distribution is defined by

$$
F(x ; \boldsymbol{\theta})=\frac{\lambda}{B(\gamma, \eta)} \int_{0}^{1-\left(1-x^{\alpha}\right)^{\beta}} y^{\gamma \lambda-1}\left(1-y^{\lambda}\right)^{\eta-1} d y,
$$

where $\boldsymbol{\theta}=(\alpha, \beta, \gamma, \eta, \lambda)^{\top}$ is the parameter vector, $\boldsymbol{\theta} \in \mathbb{R}^{+5}$. The pdf associated with (6) (for $0<x<1$ ) is obtained from (2) as

$$
f(x ; \boldsymbol{\theta})=\frac{\lambda \alpha \beta}{B(\gamma, \eta)} x^{\alpha-1}\left(1-x^{\alpha}\right)^{\beta-1}\left[1-\left(1-x^{\alpha}\right)^{\beta}\right]^{\gamma \lambda-1}\left\{1-\left[1-\left(1-x^{\alpha}\right)^{\beta}\right]^{\lambda}\right\}^{\eta-1} .
$$

Henceforth, we denote by $X \sim \operatorname{EKw}(\alpha, \beta, \gamma, \eta, \lambda)$ a random variable having the pdf (7).

Remark 2 We can see easily that the EKw distribution in (7) in non-identified when $\gamma \lambda=1$ or $\beta \eta=1$. Parameter values sometimes cannot be determined or known perfectly even in the most favorable situation, where the maximum amount of information is available, i.e. when the true distribution $f(x ; \cdot)$ is known. This problem has been known as identification problems. A parameter value $\theta_{1}$ is said to be identified if there does not exist another parameter value $\theta_{2}$ of $\boldsymbol{\theta}$ such that $f\left(x ; \theta_{1}\right)=f\left(x ; \theta_{2}\right)$. On the other hand, a parametric distribution is said to be identified if all parameter values are identified. Usually, imposing constraints on the parameters can solve some identification problems. Such constraints are said to be identifying. Thereby, to avoid identification problems in (7), we constraints the join product $\gamma \lambda$ or $\beta \eta$ different from one; $\gamma \lambda \neq 1$ or $\beta \eta \neq 1$. In addition, we can comment that any model nested in an identified model is also identified. But a nested model can be identified without that the wider model being identified.

A motivation for (6) comes from the beta construction given by Eugene et al. (2002), in which

$$
F(x ; \boldsymbol{\theta})=I_{\left[1-\left(1-x^{\alpha}\right)^{\beta}\right]^{\lambda}}(\gamma, \eta),
$$

where $I_{x}(a, b)=[B(a, b)]^{-1} \int_{0}^{x} \omega^{a-1}(1-\omega)^{b-1} d \omega$ denotes the incomplete beta function ratio. Thus, the EKw distribution can be generated by applying the beta construction (8) to a new exponentiated Kumaraswamy (ExpKw) distribution with 
parameter $\lambda$; i.e., $G_{1}(x ; \alpha, \beta, \lambda)=\left[1-\left(1-x^{\alpha}\right)^{\beta}\right]^{\lambda}$. Therefore, the EKw distribution can also be referred to as the beta exponentiated Kumaraswamy (BExpKw) distribution. For both $\gamma$ and $\eta$ positive integers, the new density becomes the pdf of the $\gamma$ th order statistic from the ExpKw distribution in a sample of size $\gamma+\eta-1$. If both $\gamma$ and $\eta$ are positive parameters on the real line, equation (8) can also be described as a simple extension of a collection of order statistics densities associated with the ExpKw distribution.

By inverting (8), we can immediately generate $X$ having the EKw distribution by $X=\left[1-\left(1-V^{1 / \lambda}\right)^{1 / \beta}\right]^{1 / \alpha}$, where $V$ is a beta random variable with parameters $\gamma$ and $\eta$. This scheme is useful because of the existence of fast generators for beta random variables. In Figures 1 and 2, we plot some possible shapes of the density function (7). It can take various forms-bathtub, $\mathrm{J}$, inverted $\mathrm{J}$, monotonically increasing or decreasing, and upside-down bathtub-depending on the parameter values. Moreover, the quantile function (qf) $Q_{E K w}(u)$ of the EKw distribution can be determined using the beta qf (say $Q_{B}(u)$ ), by $Q_{E K w}(u)=\left\{1-\left[1-Q_{B}(u)^{1 / \lambda}\right]^{1 / \beta}\right\}^{1 / \alpha}$.

\section{Special Models}

The EKw distribution is very flexible and includes several important distributions as the following eight special models.

- For $\alpha=\beta=1$, we obtain the McDonald (Mc or GB1) distribution (5) with parameters $\gamma, \eta$, and $\lambda$.

- If $\alpha=\beta=\lambda=1$, the EKw distribution reduces to the beta ( $\mathfrak{B})$ distribution with parameters $\gamma$ and $\eta$.

- If $\lambda=1$, equation (7) gives

$$
f(x ; \alpha, \beta, \gamma, \eta, 1)=\frac{\alpha \beta}{B(\gamma, \eta)} x^{\alpha-1}\left(1-x^{\alpha}\right)^{\beta \eta-1}\left[1-\left(1-x^{\alpha}\right)^{\beta}\right]^{\gamma-1} .
$$

This distribution can be viewed as a four-parameter extension of the Kw distribution. We refer to it as the beta Kumaraswamy $\mathfrak{B K w}$ distribution, since its density function can be obtained from (2) by taking $G_{1}(x ; \omega)$ as the $\mathrm{Kw}(\alpha, \beta)$ cdf and $g_{2}(x ; \boldsymbol{\tau})$ as the $\mathfrak{B}(\gamma, \eta)$ density function.

- For $\gamma=1$, (7) reduces to

$$
f(x ; \alpha, \beta, 1, \eta, \lambda)=\lambda \alpha \beta \eta x^{\alpha-1}\left(1-x^{\alpha}\right)^{\beta-1}\left[1-\left(1-x^{\alpha}\right)^{\beta}\right]^{\lambda-1}\left\{1-\left[1-\left(1-x^{\alpha}\right)^{\beta}\right]^{\lambda}\right\}^{\eta-1} .
$$

This distribution is a four-parameter generalization of the Kw distribution, that we defined as KumaraswamyKumaraswamy (KwKw) distribution. It can be obtained from (2) by replacing $G_{1}(x ; \omega)$ by the cdf of the $\mathrm{Kw}(\alpha, \beta)$ distribution and $g_{2}(x ; \tau)$ by the pdf of the $\operatorname{Kw}(\gamma, \eta)$ distribution. Its cdf has a closed-form given by

$$
F(x ; \alpha, \beta, 1, \eta, \lambda)=1-\left\{1-\left[1-\left(1-x^{\alpha}\right)^{\beta}\right]^{\lambda}\right\}^{\eta} .
$$

- If $\eta=1$ and $\gamma=1$, we have exponential Kumaraswamy (ExpKw) distribution, from (7),

$$
f(x ; \alpha, \beta, 1,1, \lambda)=\lambda \alpha \beta x^{\alpha-1}\left(1-x^{\alpha}\right)^{\beta-1}\left[1-\left(1-x^{\alpha}\right)^{\beta}\right]^{\lambda-1} .
$$

Its associated cdf is given by

$$
F(x ; \alpha, \beta, 1,1, \lambda)=G_{1}(x ; \alpha, \beta)^{\lambda},
$$

where $G_{1}(x ; \alpha, \beta)$ is the cdf of the $\operatorname{Kw}(\alpha, \beta)$ distribution. It was defined before as the three-parameter extension of the Kw distribution. If $\lambda=1$ in the ExpKw distribution, we obtain the identified nested model given by (4).

- For $\alpha=1$ and $\beta=1$, (7) reduces to beta power ( $\mathfrak{B P}$ ) distribution, with pdf as

$$
f(x ; 1,1, \gamma, \eta, \lambda)=\frac{\lambda}{B(\gamma, \eta)} x^{\gamma \lambda-1}\left(1-x^{\lambda}\right)^{\eta-1} .
$$

This density function follows from (2) if $G_{1}(x)=x^{\lambda}$ and $g_{2}(x)$ has the beta density with parameters $\gamma$ and $\eta$.

Additionally, we provide two properties of the EKw distribution.

1. If $X \sim \operatorname{EKw}(1, \beta, \gamma, \eta, \lambda)$, then $Y=X^{1 / \alpha} \sim \operatorname{GKw}(\alpha, \beta, \gamma, \eta, \lambda)$ for $\alpha>0$,

2. Let $X \sim \operatorname{EKw}(\alpha, \beta, \gamma, \eta, \lambda)$ and $Y=-\log (X)$. The pdf of $Y$ (for $y>0$ ) is given by

$$
f(y ; \boldsymbol{\theta})=\frac{\lambda \alpha \beta}{B(\gamma, \eta)} e^{-\alpha y}\left(1-e^{-\alpha y}\right)^{\beta-1}\left[1-\left(1-e^{-\alpha y}\right)^{\beta}\right]^{\gamma \lambda-1}\left\{1-\left[1-\left(1-e^{-\alpha y}\right)^{\beta}\right]^{\lambda}\right\}^{\eta-1} .
$$




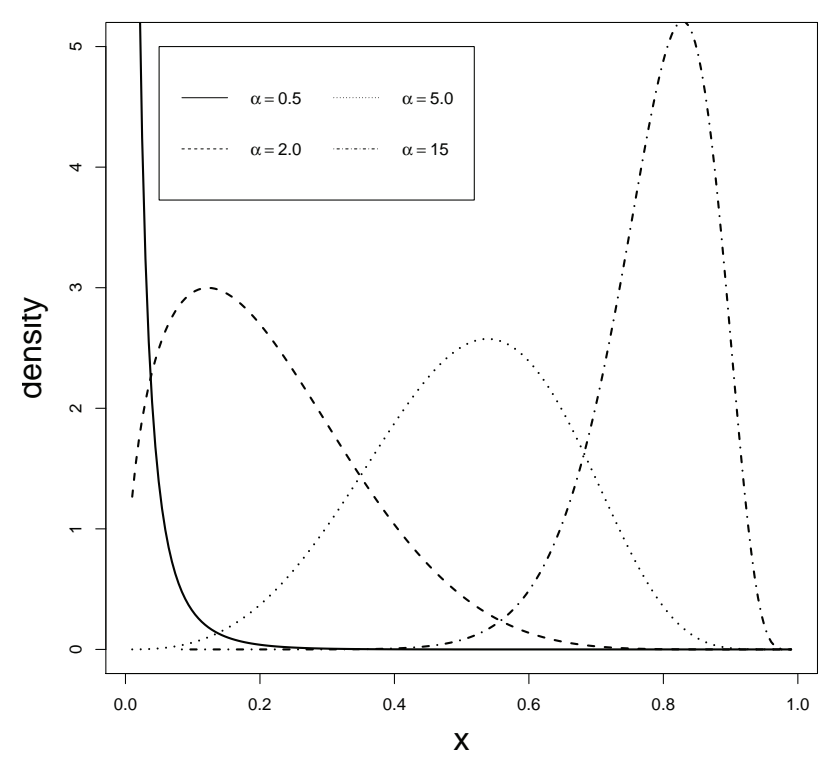

(a)

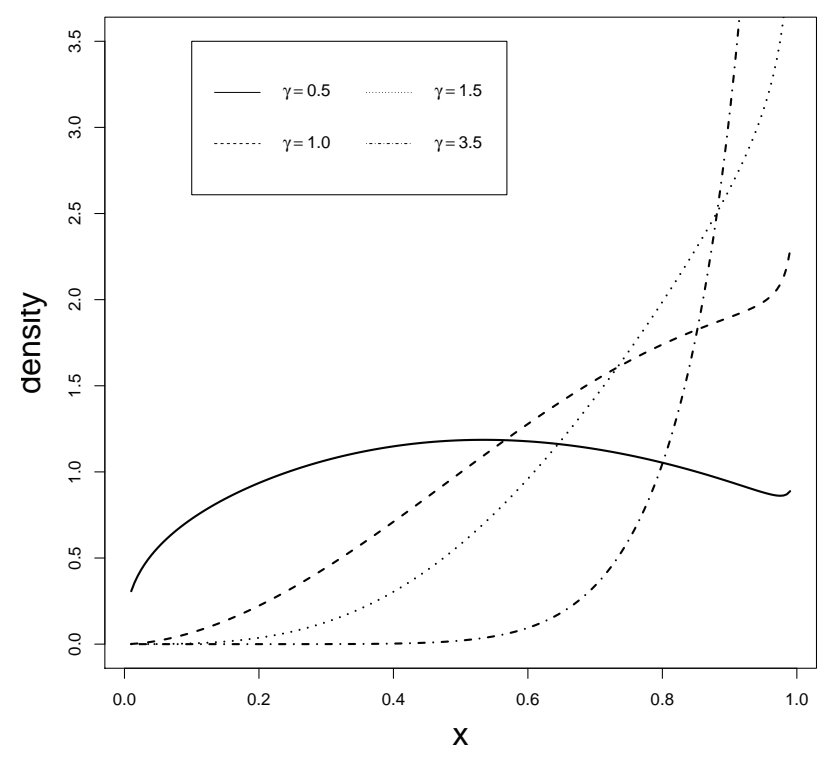

(c)

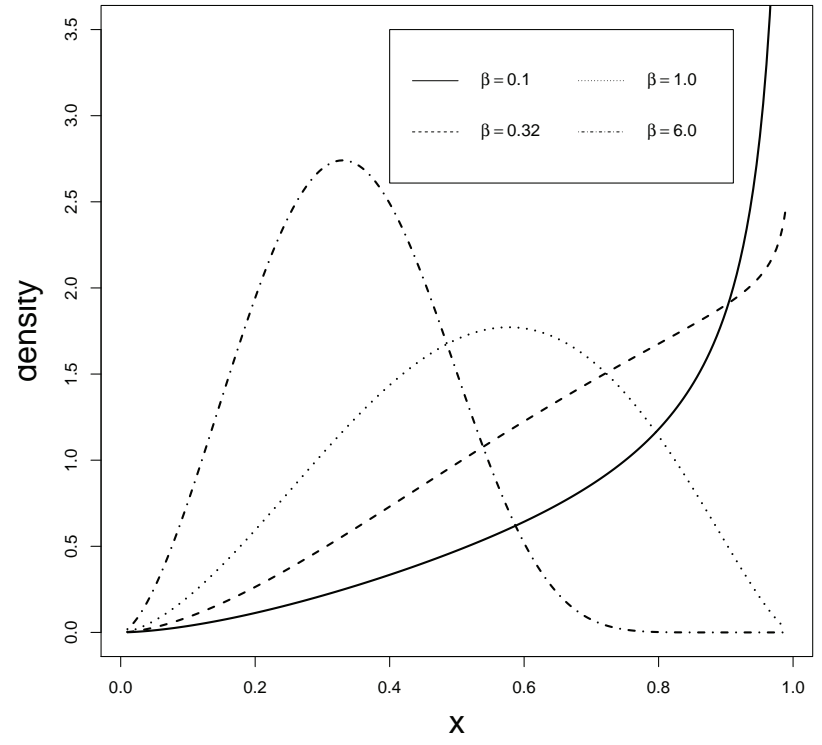

(b)

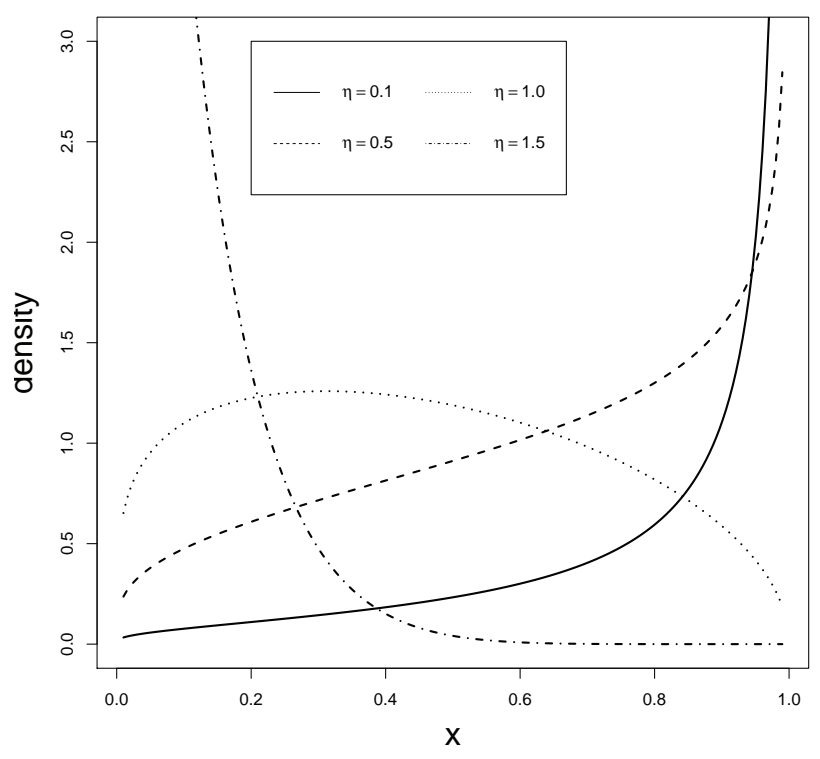

(d)

Figure 1. The EKw density functions. (a) $\boldsymbol{\theta}^{\top}=(\alpha, 3.5,1.5,2.5,0.5)^{\top}$, (b) $\boldsymbol{\theta}^{\top}=(3.5, \beta, 1.5,2.5,0.5)^{\top}$, (c) $\boldsymbol{\theta}^{\top}=(5.5,0.3, \gamma, 2.5,0.5)^{\top}$, (d) $\boldsymbol{\theta}^{\top}=(1.0,1.5,2.5, \eta, 0.5)^{\top}$, 


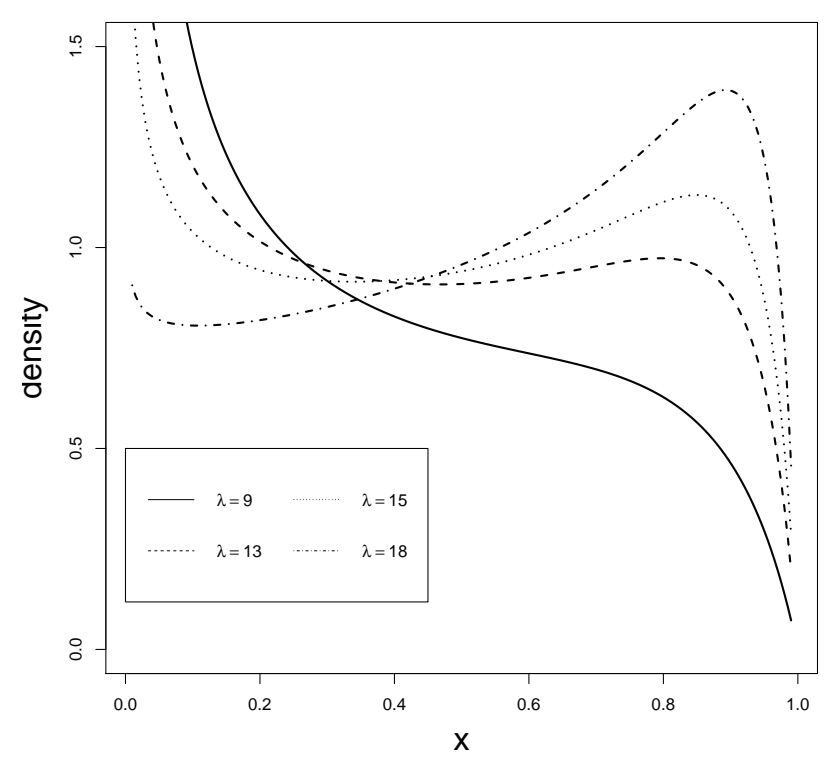

(a)

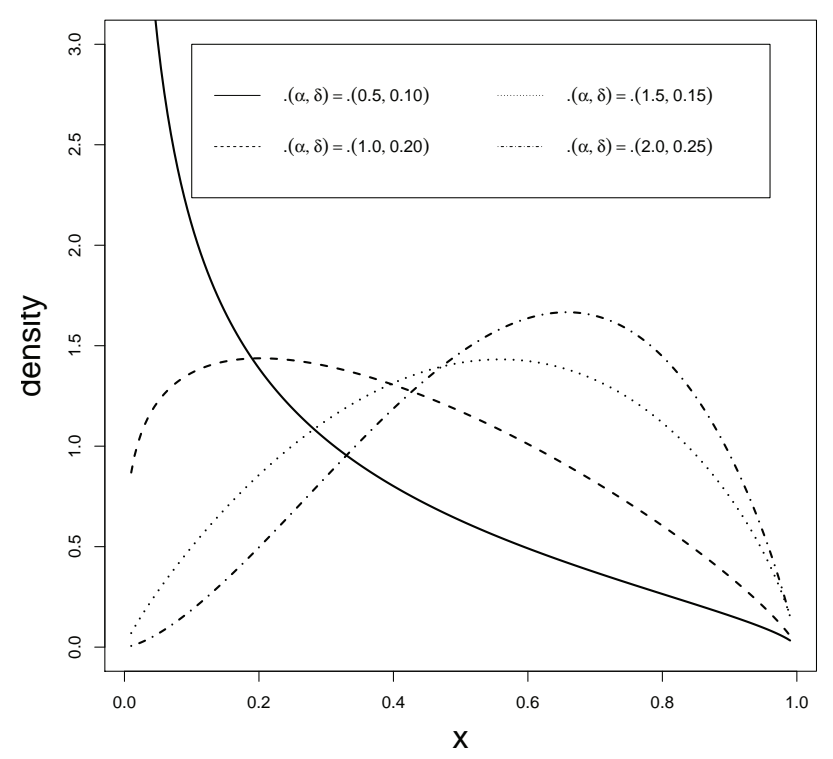

(c)

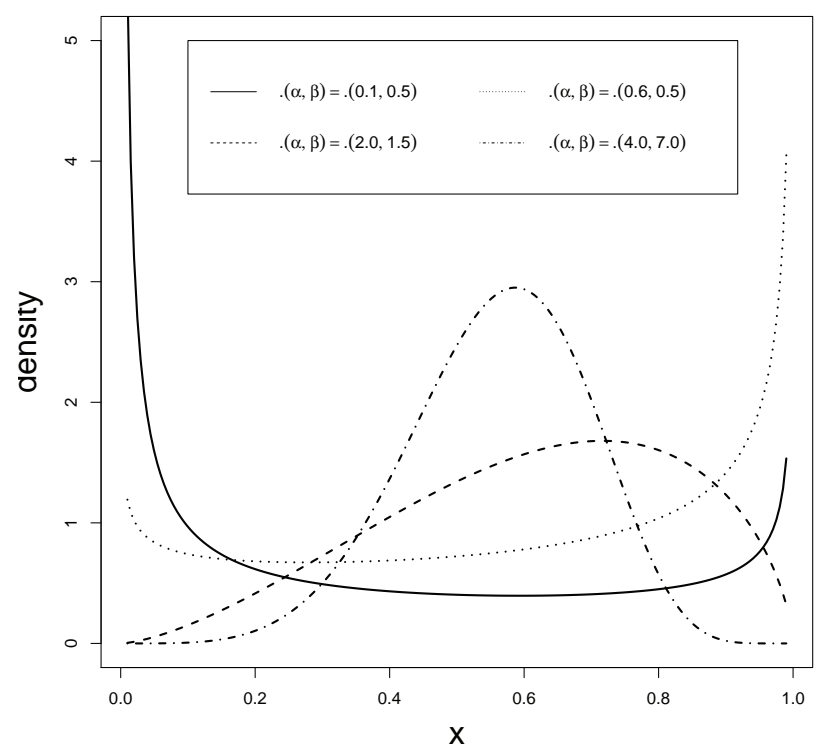

(b)

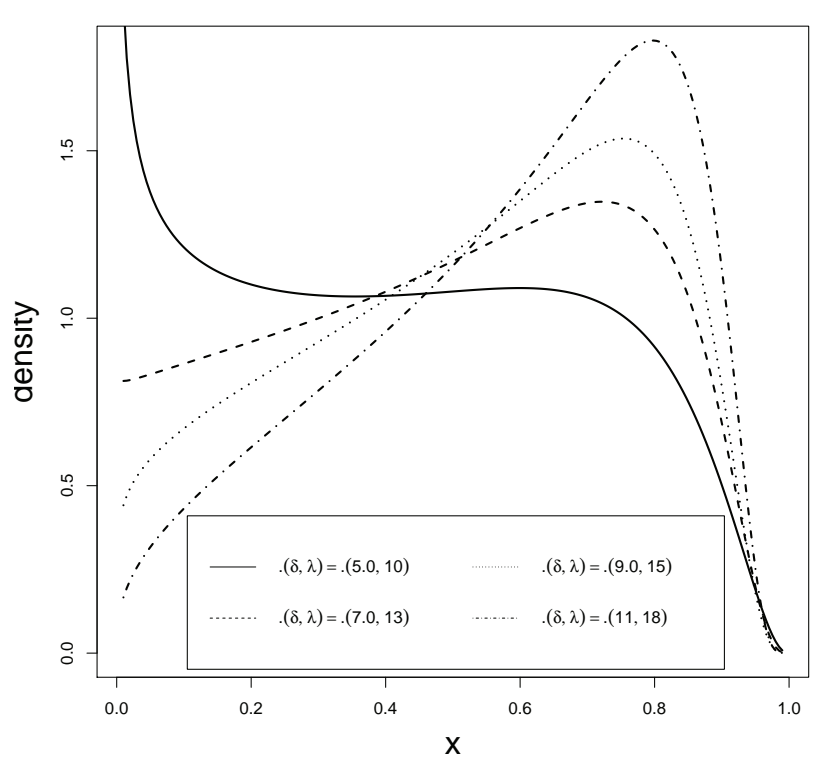

(d)

Figure 2. The EKw density functions. (a) $\boldsymbol{\theta}^{\top}=(0.5,0.7,0.1,3.0, \lambda)^{\top}$, (b) $\boldsymbol{\theta}^{\top}=(\alpha, \beta, 2.5,1.1,0.5)^{\top}$, (c) $\boldsymbol{\theta}^{\top}=(\alpha, 1.5,2.5, \eta, 0.5)^{\top}$, (d) $\boldsymbol{\theta}^{\top}=(0.5,0.7,0.15, \eta, \lambda)^{\top}$. 
We refer to (9) as the log-generalized Kumaraswamy (LEKw) distribution. The LEKw distribution is a new model that can be suitable for fitting continuous positive lifetime data. It can then be useful in survival analysis and reliability studies. The LGKw model (9) contains as special models the following two distributions.

- For $\lambda=1$, (9) reduces to beta generalized exponential ( $\mathfrak{B G E}$ ) distribution, with pdf by

$$
f(y ; \alpha, 1, \gamma, \eta, \lambda)=\frac{\alpha \beta}{B(\gamma, \eta)} e^{-\alpha y}\left(1-e^{-\alpha y}\right)^{\beta \eta-1}\left[1-\left(1-e^{-\alpha y}\right)^{\beta}\right]^{\gamma-1}, y>0 .
$$

Equation (10) is the $\mathfrak{B G E}$ density function Barreto-Souza et al. (2010). If $\gamma=1$ and $\eta=1$ in addition to $\lambda=1$, the LEKw distribution becomes the generalized exponential distribution (Gupta \& Kundu,1999). If $\lambda=\beta=\gamma=1$ and $\eta=1$, it coincides with the exponential distribution with mean $\alpha^{-1}$;

- For $\beta=1$ and $\lambda=1$, (9) reduces to beta exponential ( $\mathfrak{B E}$ ) distribution, in which

$$
f(y ; \alpha, 1, \gamma, \eta, 1)=\frac{\alpha}{B(\gamma, \eta)} e^{-\alpha \gamma y}\left(1-e^{-\alpha y}\right)^{\eta-1}, y>0
$$

which is the $\mathfrak{B E}$ density function (Nadarajah \& Kotz, 2006).

\section{Expansions}

We give a simple expansions for the cdf and pdf of the EKw distribution. If $|z|<1$ and $\eta>0$ is a non-integer real number, we have

$$
(1-z)^{\eta-1}=\sum_{j=0}^{\infty}(-1)^{j}(\eta)_{j} z^{j}
$$

where $(\eta)_{j}=[(\eta-1)(\eta-2) \ldots(\eta-j+1)] / j$ ! (for $\left.j=0,1, \ldots\right)$ denotes from now on the descending factorial. Clearly, if $(\eta)$ is a positive integer, the power series stops at $j=\eta-1$. Expanding the binomial in (6) as in equation (11), and if $\eta$ is a non-integer real number, we obtain

$$
F(x ; \boldsymbol{\theta})=\frac{\lambda}{B(\gamma, \eta)} \int_{0}^{G_{1}(x ; \alpha, \beta)} y^{\gamma \lambda-1} \sum_{j=0}^{\infty}(-1)^{j}(\eta)_{j} y^{\lambda j} d y .
$$

By simple integration, we obtain

$$
F(x ; \boldsymbol{\theta})=\sum_{j=0}^{\infty} \omega_{j} G_{1}(x ; \alpha, \beta)^{\lambda(\gamma+j)},
$$

where $\omega_{j}=\left[(-1)^{j}(\eta)_{j}\right] /[(\gamma+j) B(\gamma, \eta)]$ and $G_{1}(x ; \alpha, \beta)$ follows (3). If $\eta$ is a positive integer, the above sum stops at $j=\eta$. By differentiating (12), the pdf of $X$ follows as

$$
f(x ; \boldsymbol{\theta})=\sum_{j=0}^{\infty} \omega_{j} \lambda(\gamma+j) g_{1}(x ; \alpha, \beta) G_{1}(x ; \alpha, \beta)^{\lambda(\gamma+j)-1} .
$$

Replacing $G_{1}(x ; \alpha, \beta)$ by (3) and using (4), we have

$$
f(x ; \boldsymbol{\theta})=\sum_{k=0}^{\infty} p_{k} g_{1}(x ; \alpha,(k+1) \beta),
$$

here, $p_{k}=\sum_{j=0}^{\infty} \omega_{j} t_{j, k}, t_{j, k}=(-1)^{k} \lambda(\gamma+j)\left(\phi_{j}\right)_{k} /(k+1), \phi_{j}=(\gamma+j) \lambda-1$, and $g_{1}(x ; \alpha,(k+1) \beta) \operatorname{denotes}$ the $\operatorname{Kw}(\alpha,(k+1) \beta)$ density function with parameters $\alpha$ and $(k+1) \beta$. Further, we can express (13) as a linear combination of power density functions, since the Kw density function (4) can also be written as a linear combination of power densities. Then, we obtain

$$
f(x ; \boldsymbol{\theta})=\sum_{i=0}^{\infty} v_{i} x^{(i+1) \alpha-1},
$$

where

$$
v_{i}=(-1)^{i} \alpha \beta \sum_{k=0}^{\infty}(k+1)((k+1) \beta-1)_{i} p_{k} .
$$


Equations (13) and (14) are the main results of this section. We can obtain some mathematical properties of the EKw distribution from those of the Kw and power distributions.

\section{Moments and Generating Function}

First, we obtain an infinite linear representation for the $r$ th ordinary moment of $X$, say $\mu_{r}^{\prime}=E\left(X^{r}\right)$, from (13) as

$$
\mu_{r}^{\prime}=\sum_{k=0}^{\infty} p_{k} \tau_{r}(k)
$$

where $\tau_{r}(k)=\int_{0}^{1} x^{r} g_{1}(x ; \alpha,(k+1) \beta) d x$ is the $r$ th moment of the $\operatorname{Kw}(\alpha,(k+1) \beta)$ distribution that exists for all $r>-\alpha$. By a result of (Jones, 2009, Section 3), we have

$$
\tau_{r}(k)=(k+1) \beta B\left(1+r \alpha^{-1},(k+1) \beta\right) .
$$

Hence, the moments of $X$ are obtained directly from (15) and (16). The central moments $\left(\mu_{r}\right)$ and cumulants $\left(\kappa_{r}\right)$ of $X$ come from the ordinary moments as

$$
\mu_{r}=\sum_{k=0}^{r}(-1)^{k}\left(\begin{array}{l}
r \\
k
\end{array}\right) \mu_{1}^{\prime r} \mu_{r-k}^{\prime} \text { and } \kappa_{r}=\mu_{r}^{\prime}-\sum_{k=1}^{r-1}\left(\begin{array}{l}
r-1 \\
k-1
\end{array}\right) \kappa_{k} \mu_{r-k}^{\prime},
$$

respectively, where $\kappa_{1}=\mu_{1}^{\prime}$. So, $\kappa_{2}=\mu_{2}^{\prime}-\mu_{1}^{\prime 2}, \kappa_{3}=\mu_{3}^{\prime}-3 \mu_{2}^{\prime} \mu_{1}^{\prime}+2 \mu_{1}^{\prime 3}$, etc.

The $r$ th incomplete moment of $X$ given by $J_{r}(a ; \boldsymbol{\theta})=\int_{0}^{a} x^{r} f(x ; \boldsymbol{\theta}) d x$ is determined from the density expansion (14) as

$$
J_{r}(a ; \boldsymbol{\theta})=\sum_{i=0}^{\infty} \frac{v_{i} a^{(i+1) \alpha+r}}{(i+1) \alpha+r} .
$$

The main applications of the first incomplete moment of $X$ refer to the mean deviations, Bonferroni and Lorenz curves. We obtain the mean deviations about the mean $\mu_{1}^{\prime}=E(X)$ and about the median $M=Q_{E K w}(0.5)$ of $X$ from the relationships

$$
\delta_{1}=2\left[\mu_{1}^{\prime} F\left(\mu_{1}^{\prime} ; \boldsymbol{\theta}\right)-J_{1}\left(\mu_{1}^{\prime} ; \boldsymbol{\theta}\right)\right] \quad \text { and } \quad \delta_{2}=\mu_{1}^{\prime}-2 J_{1}(M ; \boldsymbol{\theta}),
$$

respectively. The solution of the nonlinear equation $I_{\left[1-\left(1-M^{\alpha}\right)^{\beta}\right]^{\lambda}}(\gamma, \eta)=1 / 2$ from (8) gives the median $M$. Here, $J_{1}(a ; \boldsymbol{\theta})$ follows from (17) with $r=1$.

The Bonferroni and Lorenz curves are defined by $B(\pi ; \boldsymbol{\theta})=J(q ; \boldsymbol{\theta}) /\left(\pi \mu_{1}^{\prime}\right)$ and $L(\pi ; \boldsymbol{\theta})=J_{1}(q ; \boldsymbol{\theta}) / \mu_{1}^{\prime}$, respectively, where $q=Q_{G K w}(\pi)$ is computed for a given probability $\pi$. The curves $L(\pi)$ and $B(\pi)$ for the new distribution as functions of $\pi$ are readily calculated from $J(q ; \boldsymbol{\theta})$. Additionally, the moment generating function (mgf) of the EKw distribution, say $M(t)$, follows from (14) by changing variables

$$
M(t)=\sum_{i=0}^{\infty} v_{i} t^{-(i+1) \alpha} \int_{0}^{t} u^{(i+1) \alpha-1} \exp (-u) d u .
$$

Hence, $M(t)$ reduces to the linear combination

$$
M(t)=\sum_{i=0}^{\infty} v_{i} t^{-(i+1) \alpha} \gamma((i+1) \alpha, t),
$$

where $\gamma(a, x)=\int_{0}^{a} u^{a-1} \mathrm{e}^{-\mathrm{u}} \mathrm{du}$ denotes the incomplete gamma function.

\section{Moments of Order Statistics}

The density function of the $i$ th order statistic $X_{i: n}$, say $f_{i: n}(x ; \boldsymbol{\theta})$ (for $i=1, \cdots, n$ ), in a random sample of size $n$ from the EKw distribution, follows from the binomial expansion and integration of (14) as

$$
f_{i: n}(x ; \boldsymbol{\theta})=\frac{1}{B(i, n-i+1)}\left(\sum_{t=0}^{\infty} v_{t} x^{(t+1) \alpha-1}\right) \sum_{j=0}^{n-1}\left(\begin{array}{c}
n-1 \\
j
\end{array}\right)(-1)^{j}\left(\sum_{s=0}^{\infty} v_{s}^{\star} x^{(s+1) \alpha}\right)^{i+j-1},
$$


where $v_{s}^{\star}=[(s+1) \alpha]^{-1} v_{s}$. Consider the expansion for a power series raised to a positive integer power $p$ (Gradshteyn and Ryzhik, 2000, Section 0.314).

$$
\left(\sum_{j=0}^{\infty} w_{j} x^{j}\right)^{p}=\sum_{j=0}^{\infty} c_{j, p} x^{j}
$$

where $c_{0, p}=w_{0}^{p}$ and $c_{s, p}=\left(s w_{0}\right)^{-1} \sum_{j=1}^{s}(j p-s+j) w_{j} c_{s-j, p}$ for all $s \geq 1$. Using equation (18), we can write

$$
f_{i: n}(x ; \boldsymbol{\theta})=\frac{1}{B(i, n-i+1)} \sum_{j=0}^{n-1}\left(\begin{array}{c}
n-1 \\
j
\end{array}\right)(-1)^{j} \sum_{s, t=0}^{\infty} v_{t} e_{s, i+j-1} x^{(s+t+i+j) \alpha-1},
$$

where $e_{0, i+j-1}=v_{0}^{\star(i+j-1)}$ and (for $s \geq 1$ )

$$
e_{s, i+j-1}=\left(s v_{0}^{\star}\right)^{-1} \sum_{m=1}^{s}[m(i+j-1)-s+m] v_{m}^{\star} e_{s-m, i+j-1} .
$$

The $r$ th moment of the $i t h$ order statistic can be reduced to

$$
E\left(X_{i: n}^{r}\right)=\frac{1}{\alpha B(i, n-i+1)} \sum_{j=0}^{n-1}(-1)^{j}\left(\begin{array}{c}
n-1 \\
j
\end{array}\right) \sum_{s, t=0}^{\infty} \frac{v_{t} e_{s, i+j-1}}{(r+s+t+i+j)} .
$$

Further, we obtain another explicit expression for the moments of the EKw order statistics from a general result given by Barakat and Abdelkader (2004) applied to the independent and identically distributed case. For a distribution with pdf $f(x ; \boldsymbol{\theta})$ and $\operatorname{cdf} F(x ; \boldsymbol{\theta})$, we can write

$$
E\left(X_{i: n}^{r}\right)=r \sum_{m=n-i+1}^{n}(-1)^{m-n+i-1}\left(\begin{array}{c}
m-1 \\
n-i
\end{array}\right)\left(\begin{array}{l}
n \\
m
\end{array}\right) I_{m}(r)
$$

where $I_{m}(r)=\int_{0}^{1} x^{r-1}\{1-F(x ; \boldsymbol{\theta})\}^{m} d x$. For a positive integer $m$, we have

$$
I_{m}(r)=\int_{0}^{1} x^{r-1} \sum_{p=0}^{m}(-1)^{p}\left(\begin{array}{c}
m \\
p
\end{array}\right) F(x ; \boldsymbol{\theta})^{p} d x .
$$

By replacing (12) in the above equation, we can write

$$
I_{m}(r)=\sum_{p=0}^{m}(-1)^{p}\left(\begin{array}{l}
m \\
p
\end{array}\right) \int_{0}^{1} x^{r-1}\left(\sum_{j=0}^{\infty} \omega_{j} G_{1}(x ; \alpha, \beta)^{\lambda(\gamma+j)}\right)^{p} d x .
$$

Equations (18) and (19) give

$$
I_{m}(r)=\sum_{p=0}^{m}(-1)^{p}\left(\begin{array}{l}
m \\
p
\end{array}\right) \int_{0}^{1} x^{r-1} \sum_{j=0}^{\infty} c_{j, p} G_{1}(x ; \alpha, \beta)^{\lambda(\gamma+j)} d x .
$$

By replacing $G_{1}(x ; \alpha, \beta)$ by (3) and using (11), we obtain

$$
I_{m}(r)=\sum_{p=0}^{m}(-1)^{p}\left(\begin{array}{l}
m \\
p
\end{array}\right) \sum_{j, s=0}^{\infty}(-1)^{s} c_{j, p}\left(\psi_{j}\right)_{s} \int_{0}^{1} x^{r-1}\left(1-x^{\alpha}\right)^{s \beta} d x,
$$

where $\psi_{j}=\lambda(\gamma+j)$. Since $B(a / b, c)=b \int_{0}^{1} w^{a-1}\left(1-w^{b}\right)^{c-1} d w$ for $a, b, c>0$, we have

$$
I_{m}(r)=\sum_{p=0}^{m} \sum_{j, s=0}^{\infty} s_{p, j, s} B\left(r \alpha^{-1}, s \beta+1\right),
$$

where

$$
s_{p, j, s}=\frac{(-1)^{p+s} m !}{\alpha(m-p) ! p !} c_{j, p}\left(\psi_{j}\right)_{s} .
$$


Finally, $E\left(X_{i: n}^{r}\right)$ reduces to

$$
E\left(X_{i: n}^{r}\right)=r \sum_{m=n-i+1}^{n}\left\{(-1)^{m-n+i-1}\left(\begin{array}{c}
m-1 \\
n-i
\end{array}\right)\left(\begin{array}{l}
n \\
m
\end{array}\right) \sum_{p=0}^{m} \sum_{j, s=0}^{\infty} s_{p, j, s} B\left(r \alpha^{-1}, s \beta+1\right)\right\} .
$$

\section{Rényi Entropy}

The entropy of a random variable $X$ with a density function $f(x)$ is a measure of the variation of uncertainty. One of the popular entropy measures is the Rényi entropy, given by

$$
\mathcal{J}_{R}(\rho)=\frac{1}{1-\rho} \log \left[\int f^{\rho}(x) d x\right], \rho>0, \rho \neq 1 .
$$

From (14), we can write

$$
f(x ; \boldsymbol{\theta})^{\rho}=\left(\sum_{i=0}^{\infty} v_{i} x^{(i+1) \alpha-1}\right)^{\rho} .
$$

Since $f(x ; \boldsymbol{\theta})$ belongs to the unit interval, we have (for $\rho>0$ )

$$
f(x ; \boldsymbol{\theta})^{\rho}=\sum_{j=0}^{\infty} \sum_{r=0}^{j}(-1)^{j+r}\left(\begin{array}{l}
\rho \\
j
\end{array}\right)\left(\begin{array}{l}
j \\
r
\end{array}\right) x^{(\alpha-1) r}\left(\sum_{i=0}^{\infty} v_{i} x^{i \alpha}\right)^{r} .
$$

Using equation (18), we obtain

$$
f(x ; \boldsymbol{\theta})^{\rho}=\sum_{i, j=0}^{\infty} \sum_{r=0}^{j}(-1)^{j+r}\left(\begin{array}{l}
\rho \\
j
\end{array}\right)\left(\begin{array}{l}
j \\
r
\end{array}\right) d_{i, r} x^{(i+r) \alpha-r},
$$

where $d_{0, r}=v_{0}^{r}$ and $d_{s, r}=\left(s v_{0}\right)^{-1} \sum_{m=1}^{s}(m r-s+m) v_{m} d_{s-m, r}$ (for $\left.s \geq 1\right)$. Hence,

$$
\mathcal{J}_{R}(\rho)=\frac{1}{1-\rho} \log \left[\sum_{i, j=0}^{\infty} \sum_{r=0}^{j} \frac{(-1)^{j+r}\left(\begin{array}{l}
\rho \\
j
\end{array}\right)\left(\begin{array}{l}
j \\
r
\end{array}\right) d_{i, r}}{(i+r) \alpha-r+1}\right] .
$$

\section{Estimation}

A simple method for estimating the parameters of a distribution based on a random sample is the method of moments. However, it does not work for the EKw distribution, since its moments can only be expressed in terms of the parameters as infinite sums; see Section 5. We then focus on maximum likelihood estimation. Let $x_{1}, \ldots, x_{n}$ be a random sample from the $\operatorname{EKw}(\boldsymbol{\theta})$ distribution, where $\boldsymbol{\theta}=(\alpha, \beta, \gamma, \eta, \lambda)^{\top}$ is the model parameter vector. From (7), the log-likelihood function reduces to

$$
\begin{gathered}
\ell(\boldsymbol{\theta})=n \log (\lambda)+n \log (\alpha)+n \log (\beta)-n \log [B(\gamma, \eta)]+(\alpha-1) \sum_{i=1}^{n} \log \left(x_{i}\right)+(\beta-1) \sum_{i=1}^{n} \log \left(1-x_{i}^{\alpha}\right)+ \\
(\gamma \lambda-1) \sum_{i=1}^{n} \log \left[1-\left(1-x_{i}^{\alpha}\right)^{\beta}\right]+(\eta-1) \sum_{i=1}^{n} \log \left[1-\left\{1-\left(1-x_{i}^{\alpha}\right)^{\beta}\right\}^{\lambda}\right] .
\end{gathered}
$$

The maximum likelihood estimate (MLE) $\widehat{\boldsymbol{\theta}}$ of $\boldsymbol{\theta}$ can be obtained by maximizing (20) directly by using the SAS (PROC NLMIXED), R (optim or MaxLik functions) and Ox program (sub-routine MaxBFGS). Alternatively, we can solve the nonlinear equations obtained by setting the components of the score vector $U(\boldsymbol{\theta})=\left(U_{\alpha}, U_{\beta}, U_{\gamma}, U_{\eta}, U_{\lambda}\right)$ to zero. These components are given by

$$
\begin{gathered}
U_{\alpha}(\boldsymbol{\theta})=\frac{n}{\alpha}+\sum_{i=1}^{n}\left[1-(\beta-1) z_{i}\right] \log \left(x_{i}\right)+(\gamma \lambda-1) \sum_{i=1}^{n} \frac{\dot{y}_{i(\alpha)}}{y_{i}}-(\eta-1) \lambda \sum_{i=1}^{n} v_{i} \dot{y}_{i(\alpha)}, \\
U_{\beta}(\boldsymbol{\theta})=\frac{n}{\beta}+\sum_{i=1}^{n} \log \left(1-x_{i}^{\alpha}\right)+(\gamma \lambda-1) \sum_{i=1}^{n} \frac{\dot{y}_{i(\beta)}}{y_{i}}-\lambda(\eta-1) \sum_{i=1}^{n} v_{i} \dot{y}_{i(\beta)},
\end{gathered}
$$




$$
\begin{gathered}
U_{\gamma}(\boldsymbol{\theta})=-n[\psi(\gamma)-\psi(\gamma+\eta)]+\lambda \sum_{i=1}^{n} \log \left(y_{i}\right), \\
U_{\eta}(\boldsymbol{\theta})=-n[\psi(\eta)-\psi(\gamma+\eta)]+\sum_{i=1}^{n} \log \left(1-y_{i}^{\lambda}\right), \text { and } \\
U_{\lambda}(\boldsymbol{\theta})=\frac{n}{\lambda}+\sum_{i=1}^{n}\left(\gamma-(\eta-1) y_{i} v_{i}\right) \log \left(y_{i}\right),
\end{gathered}
$$

where $\psi(\cdot)$ is the digamma function, $y_{i}=1-\left(1-x_{i}^{\alpha}\right)^{\beta}, v_{i}=y_{i}^{\lambda-1}\left(1-y_{i}^{-\lambda}\right)^{-1}, z_{i}=x_{i}^{\alpha}\left(1-x_{i}^{\alpha}\right)^{-1}, \dot{y}_{i(\alpha)}=\partial y_{i} / \partial \alpha=$ $-\beta x_{i}^{\alpha}\left(1-x_{i}^{\alpha}\right)^{\beta-1} \log \left(x_{i}\right)$, and $\dot{y}_{i(\beta)}=\partial y_{i} / \partial \beta=-\left(1-x_{i}^{\alpha}\right)^{\beta} \log \left(1-x_{i}^{\alpha}\right)$. For interval estimation and hypothesis tests on the model parameters, the observed information matrix $J=J(\theta)$ is required. Its elements are given in the Appendix.

Under standard regularity conditions, the approximate distribution of $(\widehat{\boldsymbol{\theta}}-\boldsymbol{\theta})$ is the multivariate normal $N_{5}\left(\mathbf{0}, I^{-1}(\boldsymbol{\theta})\right)$ distribution, where $I(\boldsymbol{\theta})$ is the expected information matrix. The multivariate normal $N_{5}\left(\mathbf{0}, J^{-1}(\widehat{\boldsymbol{\theta}})\right)$ distribution, where $I(\boldsymbol{\theta})$ is replaced by the estimated observed information matrix $J(\widehat{\boldsymbol{\theta}})$, is commonly used to construct approximate confidence regions. The likelihood ratio (LR) statistic can be used for testing hypotheses on the model parameters in the usual way. In particular, this statistic is useful to check if the fitted EKw distribution is statistically superior to the fitted BKw and ExpKw distributions for a given data set. For example, the test of $H_{0}: \lambda=1$ versus $H_{1}: \lambda \neq 1$ is equivalent to compare the $\mathrm{BKw}$ and $\mathrm{EKw}$ distributions and the LR statistic reduces to $w=2[\ell(\widehat{\alpha}, \widehat{\beta}, \widehat{\gamma}, \widehat{\eta}, \widehat{\lambda})-\ell(\tilde{\alpha}, \tilde{\beta}, \tilde{\gamma}, \tilde{\eta}, 1)]$, where $\widehat{\boldsymbol{\theta}}$ and $\tilde{\boldsymbol{\theta}}$ are the unrestricted and restricted estimates of $\boldsymbol{\theta}$, respectively. Under the null hypothesis, $\boldsymbol{w}$ is asymptotically distributed as $\chi_{1}^{2}$. For a given level $\zeta$, the LR test rejects $H_{0}$ if $w$ exceeds the $(1-\zeta)$ upper quantile of the $\chi_{1}^{2}$ distribution.

\section{Monte Carlo Study}

We discuss the results of a Monte Carlo simulation study conducted to assess the finite sample behavior of the MLEs. The results are obtained from 10,000 Monte Carlo simulations carried out using the subroutine BFGS implemented in the $\mathrm{R}$ software. For each replication, a random sample of size $n$ is drawn from the $\operatorname{EKw}(\alpha, \beta, \gamma, \eta, \lambda)$ distribution and the parameters are estimated by maximum likelihood. Tables 1 and 2 list the mean estimates (MEAN) of the model parameters, the corresponding biases (BIAS), the root mean squared errors (RMSE) and sample proportions delete that does not satisfy the convergence criteria $(c(\%))$ for sample sizes $n=50,100,200$ and 300. We consider two scenarios. First, we assume true parameter values $(\alpha, \beta, \gamma, \eta, \lambda)^{\top}=(3.5,50.0,45.0,33.0,50.0)^{\top}$ such that the EKw distribution is an identifiable model. Second, we assume a non-identifiable model such that the true parameters values are $(\alpha, \beta, \gamma, \eta, \lambda)^{\top}=$ $(3.5,2.0,1.0,0.5,1.0)^{\top}$, i.e., $\beta \eta=1$ and $\gamma \lambda=1$. The figures in Table 1 indicate that the biases of the MLEs become smaller for large sample size. Also, the biases, RMSEs and sample proportions deleted decrease when the sample size increases, as expected. Although, in the figures of Table 2, we can note a similar behavior, i.e. the biases, MLEs and $c(\%)$ decrease when the sample size increases; the estimators can be inconsistent, specially when samples size are small. In short, we can observed which the impact of a non-identified model can lead wrongs interpretation, given that the estimators are inconsistent.

Table 1. Simulation results for the MLEs of the EKw distributions. Identifiable model

\begin{tabular}{ccrrrrrr}
\hline $\mathrm{n}$ & Measure & $\alpha$ & $\beta$ & $\gamma$ & $\delta$ & $\lambda$ & $c(\%)$ \\
\hline \multirow{3}{*}{50} & MEAN & 3.646 & 59.323 & 48.225 & 39.676 & 52.533 & \\
& BIAS & 0.146 & 9.323 & 3.225 & 6.676 & 2.533 & 2.14 \\
& RMSE & 0.560 & 37.232 & 31.018 & 39.114 & 23.900 & \\
\hline \multirow{3}{*}{100} & MEAN & 3.594 & 55.926 & 47.297 & 35.645 & 51.673 & \\
& BIAS & 0.094 & 5.926 & 2.297 & 2.645 & 1.673 & 1.43 \\
& RMSE & 0.441 & 26.758 & 28.544 & 22.349 & 17.0340 & \\
\hline \multirow{3}{*}{200} & MEAN & 3.537 & 52.316 & 45.366 & 33.751 & 50.182 & \\
& BIAS & 0.037 & 2.316 & 0.366 & 0.7509 & 0.182 & 1.13 \\
& RMSE & 0.281 & 13.853 & 9.847 & 9.195 & 7.498 & \\
\hline \multirow{3}{*}{300} & MEAN & 3.524 & 51.548 & 45.137 & 33.555 & 50.034 & \\
& BIAS & 0.024 & 1.548 & 0.137 & 0.554 & 0.034 & 1.10 \\
& RMSE & 0.248 & 10.913 & 7.596 & 7.121 & 5.911 & \\
\hline
\end{tabular}


Table 2. Simulation results for the MLEs of the EKw distributions. Non-identifiable model

\begin{tabular}{ccrrrrrr}
\hline $\mathrm{n}$ & Measure & $\alpha$ & $\beta$ & $\gamma$ & $\delta$ & $\lambda$ & $c(\%)$ \\
\hline \multirow{3}{*}{50} & MEAN & 6.117 & 4.404 & 2.899 & 0.621 & 3.410 & \\
& BIAS & 2.617 & 2.404 & 1.899 & 0.121 & 2.410 & 116.7 \\
& RMSE & 7.301 & 3.999 & 3.708 & 2.583 & 5.293 & \\
\hline \multirow{3}{*}{100} & MEAN & 5.708 & 3.755 & 2.571 & 0.404 & 2.322 & \\
& BIAS & 2.208 & 1.755 & 1.571 & -0.096 & 1.322 & 89.96 \\
& RMSE & 6.196 & 2.850 & 2.998 & 0.451 & 3.247 & \\
\hline \multirow{2}{*}{200} & MEAN & 5.538 & 3.339 & 2.375 & 0.410 & 1.722 & \\
& BIAS & 2.038 & 1.339 & 1.375 & -0.090 & 0.722 & 66.69 \\
& RMSE & 5.359 & 2.235 & 2.688 & 0.229 & 2.118 & \\
\hline \multirow{3}{*}{300} & MEAN & 5.368 & 3.143 & 2.280 & 0.417 & 1.466 & \\
& BIAS & 1.868 & 1.143 & 1.280 & -0.083 & 0.466 & 54.82 \\
& RMSE & 4.808 & 1.913 & 2.576 & 0.215 & 1.662 & \\
\hline
\end{tabular}

\section{Application}

We present an application of the EKw distribution to the observed percentages of children living in households with a per capita income of less than R $\$ 75.50$ in 1991 in 5507 Brazilian municipal districts. The data set was extracted from the Atlas of Brazil Human Development available at http://www.pnud.org.br/. The EKw distribution includes some important sub-models (as described in Section 3 that allow their evaluation relative to it. Table 3 lists the MLEs and their standard errors for some fitted models to the current data. The histogram of these data and the estimated densities of the fitted models are displayed in Figure 3. It is clear from these plots that the EKw distribution gives the best fit to these data.

Table 4 gives the values of the following statistics for some models: $\ell(\widehat{\boldsymbol{\theta}})$, Akaike Information Criterion (AIC), Bayesian Information Criterion $(B I C)$ and a corrected criterion $\left(A I C_{c}\right)$. The figures in this table indicate that the EKw distribution is a better model for the current data. Further, we determine the maximum values of the unrestricted and restricted loglikelihoods to obtain LR statistics for testing some sub-models of the new distribution. The computations are carried out using the optim function implemented in the R software. For testing $H_{0}:(\alpha, \beta, \lambda)=(1,1,1)$ versus $H_{1}: H_{0}$ is not true, i.e., for comparing the EKw and beta models, $w=2(1514.262-1271.561)=485.402$ (p-value $<0.001)$. This indicates that the EKw model gives a better representation for the data than the beta distribution. Also, the LR statistic for comparing the EKw and BKw models, i.e., for testing $H_{0}: \lambda=1$ versus $H_{1}: \lambda \neq 1$, is $w=2(1514.262-1383.464)=261.596$ (p-value < 0.001). It also yields a favorable indication for the EKw model. The maximized log-likelihoods in Table 4 give evidence that the LR statistics for comparing the EKw distribution with any sub-model support the wider distribution as a better model for the current data.

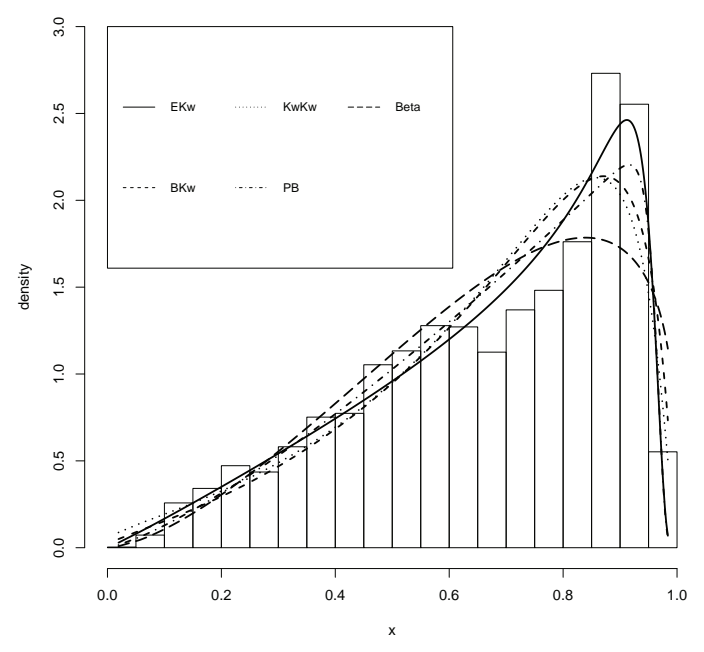

Figure 3. Histogram and estimated densities for the percentage of children living in households with a per capita income of less than R \$ 75.50 (1991) in 5507 Brazilian municipal districts. 
Table 3. MLEs of the model parameters for the percentage of children living in households with a per capita income of less than R\$ 75.50 (1991) in 5507 brazilian municipal districts.

\begin{tabular}{crrrrr}
\hline Distribution & $\alpha$ & $\beta$ & $\gamma$ & $\delta$ & $\lambda$ \\
\hline$E K w$ & 3.616 & 0.573 & 0.072 & 22.953 & 7.944 \\
& $(0.808)$ & $(0.072)$ & $(0.008)$ & $(5.746)$ & $(1.917)$ \\
$B K w$ & 0.047 & 0.063 & 18.689 & 61.113 & - \\
& $(0.012)$ & $(0.010)$ & $(2.349)$ & $(11.390)$ & \\
$K w K w$ & 0.138 & 0.386 & - & 8.010 & 6.970 \\
& $(0.076)$ & $(0.054)$ & & $(1.749)$ & $(2.580)$ \\
$B P$ & - & - & 0.032 & 11.101 & 71.635 \\
& & & $(0.003)$ & $(2.169)$ & $(6.667)$ \\
Beta & - & - & 2.568 & 1.301 & - \\
& & & $(0.048)$ & $(0.023)$ & \\
\hline
\end{tabular}

Table 4. The measures $\ell(\widehat{\boldsymbol{\theta}})$, AIC, BIC and CAIC for the the percentage of children living in households with a per capita income of less than R 75.50 (1991) in 5507 brazilian municipal districts.

\begin{tabular}{crrrr}
\hline Distribution & $\ell(\widehat{\boldsymbol{\theta}})$ & $A I C$ & $B I C$ & $A I C_{c}$ \\
\hline$E K w$ & 1514.262 & -3018.524 & -3018.524 & -3018.513 \\
$B K w$ & 1383.464 & -2758.928 & -2758.928 & -2758.921 \\
$K w K w$ & 1385.272 & -2762.544 & -2762.544 & -2762.537 \\
$B P$ & 1484.568 & -2963.136 & -2943.295 & -2963.129 \\
Beta & 1271.561 & -2539.122 & -2525.894 & -2539.118 \\
\hline
\end{tabular}

\section{Conclusions}

We propose a new five-parameter continuous distribution on the standard unit interval that generalizes the beta, Kumaraswamy (Kumaraswamy, 1980), and McDonald (McDonald, 1984) distributions and includes, as special models, other distributions discussed in the literature. We refer to the new model as the extended Kumaraswamy (EKw) distribution and study some of its structural properties. We assume some constraints such that EKw distribution is an identified model. We demonstrate that the EKw density function can be expressed as linear combinations of Kumaraswamy and power density functions. We provide explicit expressions for ordinary and incomplete moments, generating and quantile functions, mean deviations, Bonferroni and Lorenz curves, and Rényi's entropy. The density of the order statistics can also be expressed as a linear combination of power densities. We obtain explicit expressions for their moments. Parameter estimation is approached by maximum likelihood, and the performance of the estimates and the impact of identifiability problems are evaluated through a simulation study. The usefulness of the new distribution is illustrated by means of a real data set. We hope that the proposed extended model may attract wider applications in the analysis of continuous proportions data.

\section{Acknowledgements}

We gratefully acknowledge financial support from CNPq-Brazil. We also thank two anonymous reviewers for constructive comments and suggestions.

\section{References}

Barakat, H., \& Abdelkader, Y. H. (2004). Computing the moments of order statistics from nonidentical random variables. Statistical Methods and Applications, 13, 15-26. https://doi.org/10.1007/s10260-003-0068-9

Barreto-Souza, W., Santos, A. S., \& Cordeiro, G. (2010). The beta generalized exponential distribution. Journal of Statistical Computation and Simulation, 80,159-172. https://doi.org/10.1080/00949650802552402

Courard-Hauri, D. (2007). Using Monte Carlo analysis to investigate the relationship between overconsumption and uncertain access to one's personal utility function. Ecological Economics, 64, 152-162. https://doi.org/10.1016/j.ecolecon.2007.02.018

Eugene, N., Lee, C., \& Famoye, F. (2002). Beta-normal distribution and its applications. Communications in StatisticsTheory and Methods, 31, 497-512. https://doi.org/10.1081/STA-120003130

Ferreira, J. T., \& Steel, M. (2006). A constructive representation of univariate skewed distribution. Journal of the American Statistical Association, 101, 823-829. https://doi.org/10.1198/016214505000001212

Fletcher, S. C., \& Ponnambalam, K. (1996). Estimation of reservoir yield and storage distribution using moments analysis. Journal of Hydrology, 182, 259-275. https://doi.org/10.1016/0022-1694(95)02946-X 
Ganji, A., Ponnambalam, K., Khalili, D., \& Karamouz, M. (2006). Grain yield reliability analysis with crop water demand uncertainty. Stochastic Environmental Research and Risk Assessment, 20, 259-277. https://doi.org/10.1007/s00477005-0020-7

Gradshteyn, I. S., \& Ryzhik, I. M. (2000). Table of Integrals, Series, and Products.Academic Press, New York.

Gupta, A. K., \& Kundu, D. (1999). Generalized exponential distributions. Australian and New Zealand Journal of Statistics, 41, 173-188. https://doi.org/10.1111/1467-842X.00072

Gupta, A. K., \& Nadarajah, S. (2004a). Handbook of Beta Distribution and its Applications.Marcel Dekker, New York.

Gupta, A. K., \& Nadarajah, S. (2004b). On the moments of the beta normal distribution. Communications in StatisticsTheory and Methods, 33, 1-13. https://doi.org/10.1081/STA-120026573

Jones, M. C. (2009). Kumaraswamy's distributions: A beta-type distribution with some tractability advantages. Statistical Methodology, 6, 70-81. https://doi.org/10.1016/j.stamet.2008.04.001

Kumaraswamy, P. (1980). A generalized probability density function for double bounded random processes. Journal of Hydrology, 46, 79-88. https://doi.org/10.1016/0022-1694(80)90036-0

McDonald, J. B. (1984). Some generalized function for the size distributions of income. Econometrica, 52, 647-663. https://doi.org/10.2307/1913469

Nadarajah, S., \& Gupta, A. K. (2004). The beta frechet distribution. Far East Journal of Theoretical Statistics, 14, 15-24. https://doi.org/10.1155/S1024123X04403068

Nadarajah, S., \& Kotz, S. (2004). The beta gumbel distribution. Mathematical Problems in Engineering, 4, 323-332. https://doi.org/10.1007/s10440-006-9055-0

Nadarajah, S., \& Kotz, S. (2006). The exponentiated type distributions. Acta Applicandae Mathematicae, 92, 97-11.

Sanchez, S., Ancheyta, J., \& McCaffrey, W. C. (2007). Comparison of probability distribution function for fitting distillation curves of petroleum. Energy and Fuels, 21, 2955-2963. https://doi.org/10.1021/ef070003y

Seifi, A., Ponnambalam, K., \& Vlach, J. (2000). Maximization of manufacturing yield of systems with arbitrary distributions of component values. Annals of Operations Research, 99, 373-383. https://doi.org/10.1023/A:1019288220413

Sundar, V., \& Subbiah, K. (1989). Application of double bounded probability density-function for analysis of ocean waves. Ocean Engineering, 16, 193-200. https://doi.org/10.1016/0029-8018(89)90005-X

Wahed, A. S. (2006). A general method of constructing extended families of distribution from an existing continuous class. Journal of Probability and Statistical Science, 4, 165-177.

\section{Appendix}

The elements of the observed information matrix $J(\theta)$ for $(\alpha, \beta, \gamma, \eta, \lambda)$ are given by

$$
\begin{gathered}
J_{\alpha \alpha}=-\frac{n}{\alpha^{2}}-(\beta-1) \sum_{i=1}^{n} \dot{z}_{i(\alpha)} \log \left(x_{i}\right)+(\gamma \lambda-1) \sum_{i=1}^{n}\left[\frac{\ddot{y}_{i(\alpha)}}{y_{i}}-\left(\frac{\dot{y}_{i(\alpha)}}{y_{i}}\right)^{2}\right]-(\eta-1) \lambda \sum_{i=1}^{n}\left[\dot{v}_{i(\alpha)} \dot{y}_{i(\alpha)}+v_{i} \ddot{y}_{i(\alpha)}\right], \\
J_{\alpha \beta}=-\sum_{i=1}^{n} z_{i} \log \left(x_{i}\right)+(\gamma \lambda-1) \sum_{i=1}^{n}\left[\frac{\ddot{y}_{i(\alpha \beta)}}{y_{i}}-\frac{\dot{y}_{i(\alpha)} \dot{y}_{i(\beta)}}{y_{i}^{2}}\right]-(\eta-1) \lambda \sum_{i=1}^{n}\left[\dot{v}_{i(\beta)} \dot{y}_{i(\alpha)}+v_{i} \ddot{y}_{i(\alpha \beta)}\right], \\
J_{\alpha \gamma}=\lambda \sum_{i=1}^{n} \frac{\dot{y}_{i(\alpha)}}{y_{i}}, \\
J_{\alpha \beta}=-\lambda \sum_{i=1}^{n} v_{i} \dot{y}_{i(\alpha)}, \quad J_{\alpha \lambda}=\sum_{i=1}^{n}\left[\gamma / y_{i}-(\eta-1) v_{i}\right] \dot{y}_{i(\alpha)}, \\
J_{\beta \gamma}=\lambda \sum_{i=1}^{n} \frac{\dot{y}_{i(\beta)}}{\beta_{i}}, \quad J_{\beta \eta}=-\lambda \sum_{i=1}^{n} v_{i} \dot{y}_{i(\beta)}, \quad J_{\beta \lambda}=\gamma \sum_{i=1}^{n} \frac{\dot{y}_{i(\beta)}}{y_{i}}-(\eta-1) \sum_{i=1}^{n} v_{i} \dot{y}_{i(\beta)}, \\
J_{\gamma \gamma}=-n\left\{\dot{y}_{i}^{\prime}(\gamma)-\psi^{\prime}(\gamma+\eta)\right\}, \quad \dot{y}_{\gamma \eta}=n \psi^{\prime}(\gamma+\eta), \quad J_{\gamma \lambda}=\sum_{i=1}^{n} \log \left(y_{i}\right),
\end{gathered}
$$




$$
\begin{gathered}
J_{\eta \eta}=-n\left\{\psi^{\prime}(\eta)-\psi^{\prime}(\gamma+\eta)\right\}, \quad J_{\eta \lambda}=-\sum_{i=1}^{n} y_{i} v_{i} \log \left(y_{i}\right), \text { and } \\
J_{\lambda \lambda}=-\frac{n}{\lambda^{2}}-(\eta-1) \sum_{i=1}^{n} y_{i} \dot{v}_{i(\lambda)} \log \left(y_{i}\right),
\end{gathered}
$$

where $\dot{z}_{i(\alpha)}=\partial z_{i} / \partial \alpha=\left(1+z_{i}\right) z_{i} \log \left(x_{i}\right), \ddot{y}_{i(\alpha)}=\partial^{2} y_{i} / \partial \alpha^{2}=\left[1-(\beta-1) z_{i}\right] \dot{y}_{i(\alpha)} \log \left(x_{i}\right), \ddot{y}_{i(\beta)}=\partial^{2} y_{i} / \partial \beta^{2}=\dot{y}_{i(\beta)} \log \left(1-x_{i}^{\alpha}\right)$, $\ddot{y}_{i(\alpha \beta)}=\partial^{2} y_{i} / \partial \alpha \partial \beta=\left[1 / \beta+\log \left(1+x_{i}^{\alpha}\right)\right] \dot{y}_{i(\alpha)}, \dot{v}_{i(\alpha)}=\partial v_{i} / \partial \alpha=\left[(\lambda-1) / y_{i}+\lambda v_{i}\right] v_{i} \dot{y}_{i(\alpha)}, \dot{v}_{i(\beta)}=\partial v_{i} / \partial \beta=\left[(\lambda-1) / y_{i}+\lambda v_{i}\right] v_{i} \dot{y}_{i(\beta)}$, $\dot{v}_{i(\lambda)}=\partial v_{i} / \partial \lambda=\left(1+y_{i} v_{i}\right) v_{i} \log \left(y_{i}\right)$, and $\psi^{\prime}(\cdot)$ is the first derivative of the digamma function.

\section{Copyrights}

Copyright for this article is retained by the author(s), with first publication rights granted to the journal.

This is an open-access article distributed under the terms and conditions of the Creative Commons Attribution license (http://creativecommons.org/licenses/by/4.0/). 